\title{
ABORDAGEM TRANSDIAFRAGMÁTICA NO TRANSPLANTE HEPÁTICO COM DOADOR FALECIDO NA SÍNDROME DE BUDD-CHIARI: DESAFIO DA TÉCNICA CIRÚRGICA-RELATO DE CASO.
}

\author{
Transdiaphragmatic approaching in deceased donor liver transplantation for Budd-Chiari \\ Syndrome: surgical challenge-case report
}

\author{
Olival Cirilo Lucena da Fonseca Neto; Luiz Eduardo Rafael Moutinho; Priscylla Jennie Monteiro Rabêlo; \\ Paulo Sergio Vieira de Melo; Américo Gusmão Amorim; Claudio Moura Lacerda
}

\begin{abstract}
RESUMO
Introdução: Budd-Chiari é uma síndrome heterogênea de etiologia não específica, caracterizada pela obstrução da drenagem venosa hepática ao nível das veias hepáticas até o átrio direito. É encontrada com frequência nesses pacientes fibrose de etiologia desconhecida entre o diafragma e a veia cava supra-hepática, tornando a dissecação e anastomoses inviáveis nesse segmento. Objetivo: Relatar e revisar os desafios da técnica cirúrgica acerca da abordagem transdiafragmática e anastomose cavo-atrial no transplante hepático devido à oclusão completa da veia cava inferior retro-hepática. Método: Relato de caso com base em observações cirúrgicas, registros clínicos e imagens produzidos durante o acompanhamento do paciente no serviço de transplante. Dados da literatura da plataforma Pubmed embasaram a discussão. Conclusões: A abordagem transdiafragmática e anastomose cavo-atrial mostram-se como alternativas factíveis na literatura atual para pacientes com síndrome de Budd-Chiari que necessitem do transplante. A substituição da veia cava infra-hepática depende do desenvolvimento de colaterais através do sistema ázigo e deve ser avaliada durante o pré-operatório.
\end{abstract}

Descritores: Transplante Hepático; Síndrome de Budd-Chiari; Veia Cava Inferior.

Instituições:

Serviço de Cirurgia Geral e Transplante de Fígado do Hospital Universitário Oswaldo Cruz (HUOC) - UPE, Recife, Pernambuco, Brasil.

\section{Correspondência:}

Olival Cirilo Lucena Fonseca Neto.

Unidade de Transplante de Fígado - R. Arnóbio Marquês, 310 - CEP 50100-130, Recife/PE,

Tel.: (81)991626477.

E-mail: olivalneto@globo.com

Recebido em: 27/08/2019

Aceito em: 28/09//2019

\section{INTRODUÇÃO}

Budd-Chiari é uma síndrome heterogênea de etiologia não específica, caracterizada pela obstrução da drenagem venosa hepática ao nível das veias hepáticas, até o átrio direito. ${ }^{1} \mathrm{~A}$ doença pode determinar aumento da pressão sinusoidal, hipertensão portal, isquemia e necrose do parênquima hepático, culminando em regeneração hiperplásica nodular, fibrose e cirrose hepática. $^{2}$

O desenvolvimento de veias colaterais do sistema porta ou de shunt porto-sistêmico pode diminuir a pressão sinusoidal e garantir o funcionamento hepático. ${ }^{3}$ É encontrada, com frequência, nesses pacientes fibrose 
de etiologia desconhecida entre o diafragma e a veia cava supra-hepática, tornando a dissecação e anastomoses inviáveis nesse segmento. ${ }^{4}$ As alternativas cirúrgicas tornam-se, portanto, limitadas. O procedimento de Senning, ressecção dorsal do fígado com anastomose cavoatrial foi proposto, porém, com limitações. O shunt porto-sistêmico e o transplante hepático ( $\mathrm{TxH})$ têm sido as alternativas terapêuticas cirúrgicas mais utilizadas para esse grupo de pacientes. Entretanto, para garantir a drenagem venosa adequada do enxerto hepático nesse grupo de pacientes fazem-se necessárias modificações da técnica cirúrgica convencional.

\section{RELATO DO CASO}

Paciente, sexo feminino, 29 anos, com quadro crônico de edema nos membros inferiores e ascite. Foi diagnosticada com Síndrome de Budd-Chiari (SBC) de etiologia não definida e submetida ao transplante ortotópico de fígado com doador falecido pela técnica convencional, após deterioração da função hepática. A paciente apresentava MELD de 18 pontos e Child-Pugh classe $C$.

A cavografia constatou ausência de fluxo sanguíneo na veia cava retro-hepática e desenvolvimento abundante de circulação colateral através do sistema ázigo. O exame de imagem na veia porta também revelou ausência de fluxo sanguíneo. Anastomose cavo-caval términoterminal não foi possível, devido à fibrose e atrofia do segmento infra-diafragmático da veia cava. Para acessar o segmento sadio, uma janela oval na região tendinosa do diafragma foi realizada imediatamente anterior ao hiato da veia cava, proporcionando comunicação entre o peritônio e o pericárdio (figura 1).

Figura 1 - Fase anepática: clampeamento do hiato caval, clampeamento da veia cava inferior intrapericárdica e exposição da cavidade pericárdica através da janela oval

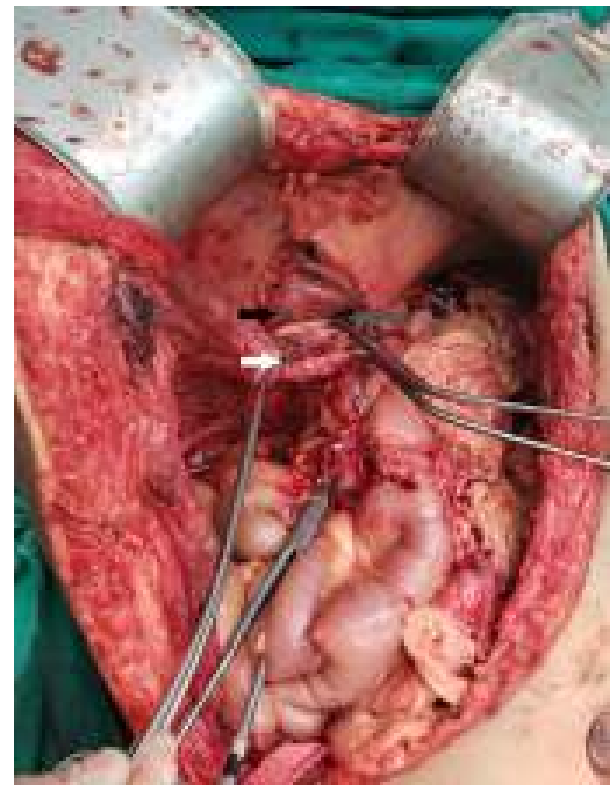

Realizada anastomose cavo-atrial (figuras 2).

Figuras 2 - Enxerto fora da solução de preservação dentro da cavidade abdominal do receptor: margem posterior da anastomose cavo-atrial concluída (esquerda). Aspecto final da anastomose cavoatrial após realizada a reperfusão retrógada (direita).

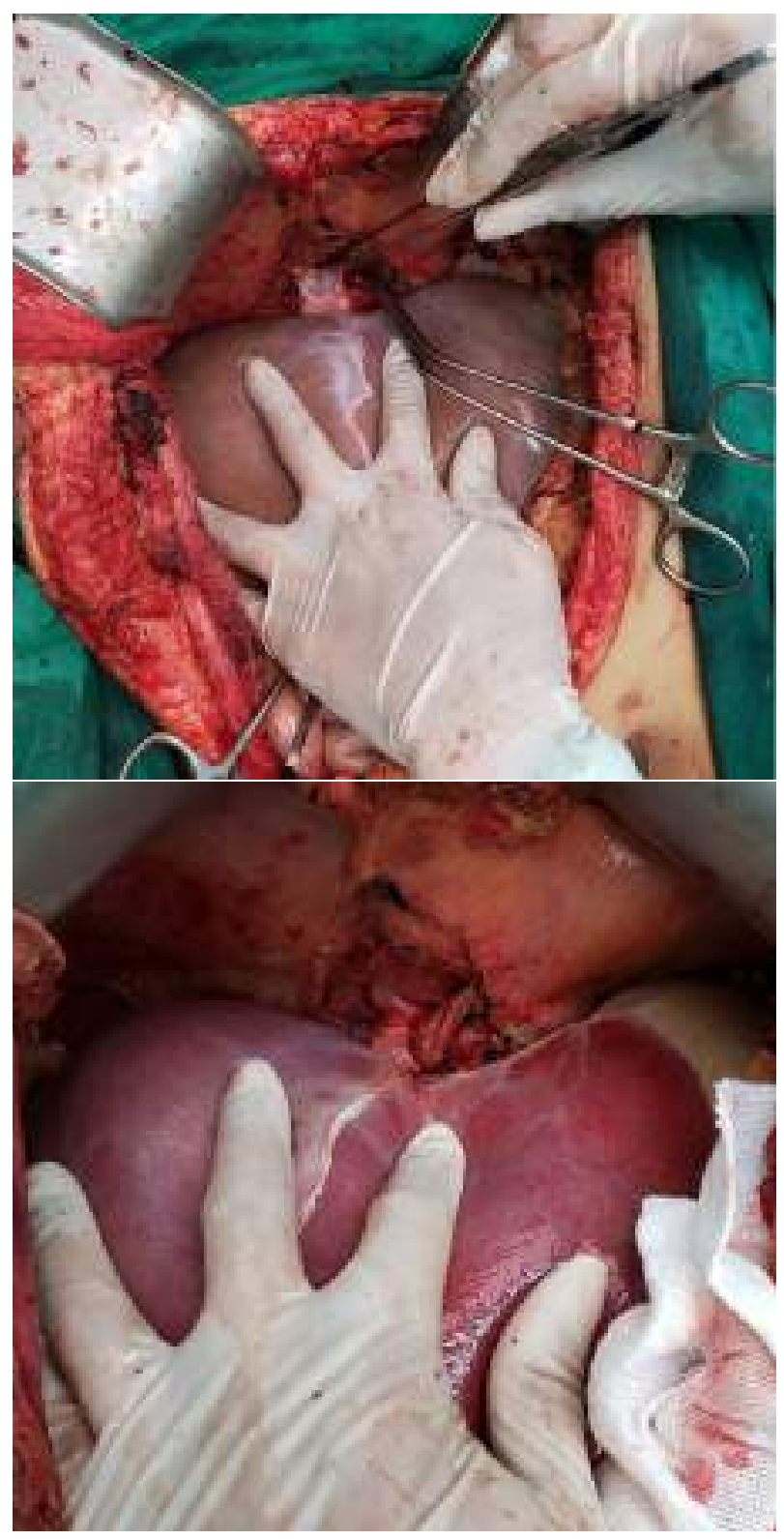

Não foi reconstruído o retorno venoso através da veia cava infra-hepática do enxerto e não foi necessária a realização de drenagem torácica. O fluxo portal foi restabelecido com anastomose término-lateral entre a veia porta do enxerto e a veia gástrica esquerda do receptor (figura 3). A cirurgia durou 6 horas e 15 minutos, a fase anepática, 1 hora e 40 minutos. O enxerto apresentou aspecto positivo após a revascularização (figura 4) e o estudo do explante confirmou os achados cirúrgicos (figura 5 ). 
Olival Cirilo Lucena da Fonseca Neto; Luiz Eduardo Rafael Moutinho; Priscylla Jennie Monteiro Rabêlo; Paulo Sergio Vieira de Melo; Américo Gusmão Amorim; Claudio Moura Lacerda

Figura 3 - Hilo hepático após concluído a revascularização do enxerto. Anastomose termino-lateral da veia porta com a veia

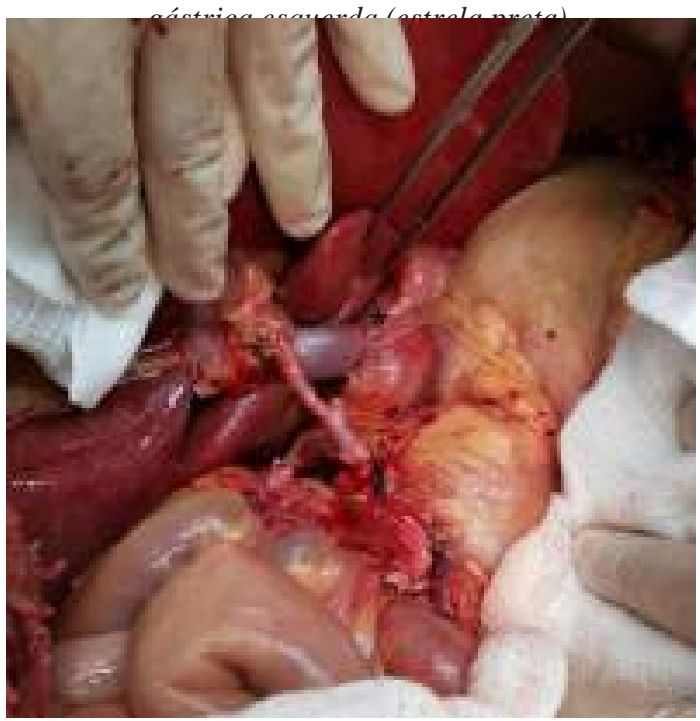

Figura 4 - Enxerto nosicionado e revascularizado na cavidade

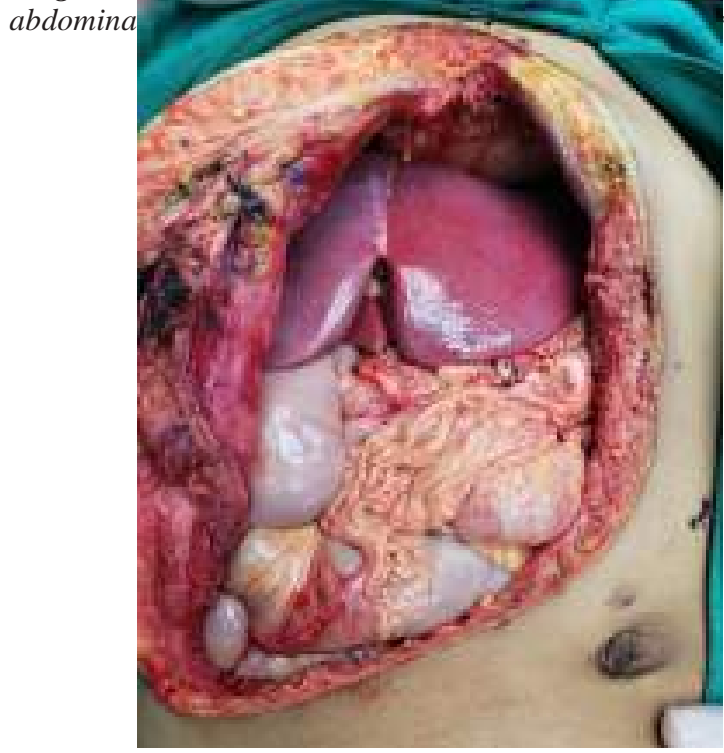
dido com

Figura 5 - Explante vista posterior/diafragmática. A pinça destaca a fibrose e atrofia da veia cava inferior.

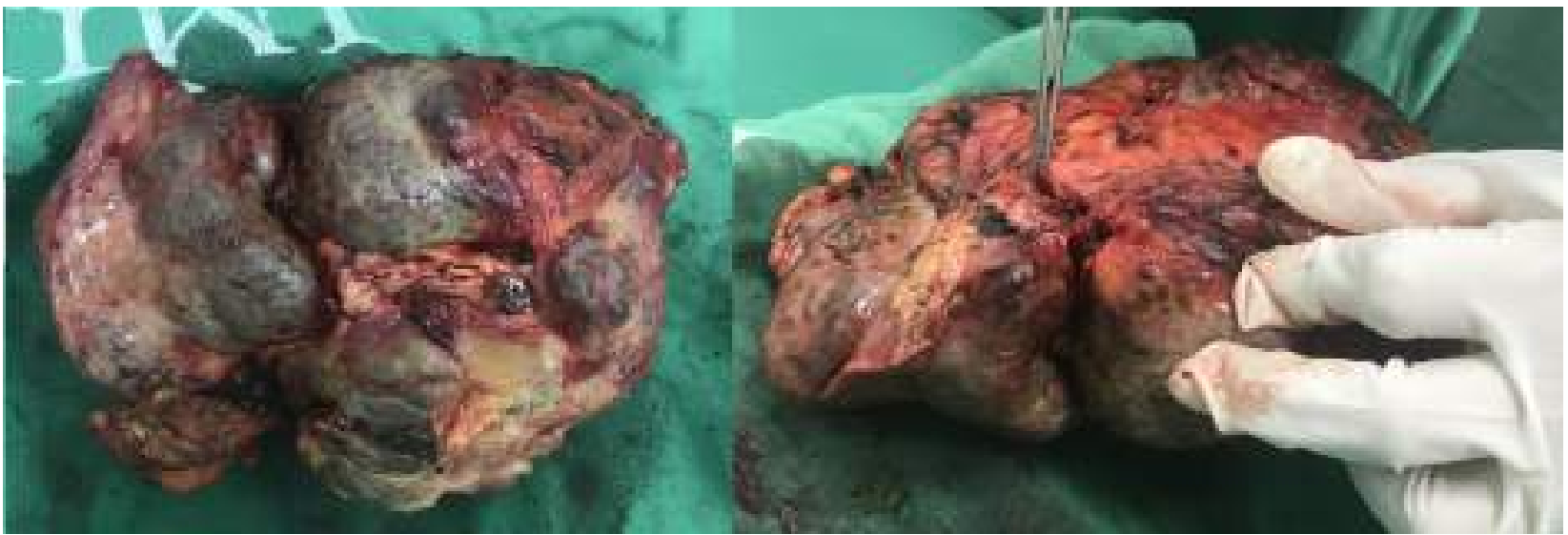

\section{DISCUSSÃO}

O tratamento da SBC consiste de uma série de medidas clínicas e cirúrgicas que progride por etapa em função do grau de complexidade e da resposta terapêutica do paciente. Segue em sequência anticoagulação, trombólise, diuréticos, angioplastia, shunt transjugular porto-sistêmico e por fim, TxH. Cerca de 10-20\% desses pacientes evoluem para insuficiência hepática e necessitam, portanto, do transplante. ${ }^{5} \mathrm{De}$ fato, o TxH é tratamento viável para os portadores da SBC, independente da condição precedente à síndrome e da complexidade do procedimento. 6 Os resultados em longo prazo o consolidam como alternativa efetiva, apesar da alta mortalidade precoce. ${ }^{6,7}$
A anastomose cavo-atrial no TxH é indicada para SBC, doença litiásica intra-hepática e equinococose alveolar incurável quando acometem ao nível diafragmático. ${ }^{8,9}$ É reportado que a trombose da veia cava inferior ( $\mathrm{VCl})$ pode estar presente em até $16 \%$ dos pacientes submetidos ao TxH devido a SBC, porém, a oclusão de todo segmento da veia é ainda mais rara. ${ }^{6}$

No que se refere à cirurgia reportada, a dissecação da face diafragmática mostrou-se desafiadora, devido ao grau de aderências próximas a $\mathrm{VCl}$. A manutenção de um segmento extenso da $\mathrm{VCl}$ do enxerto foi importante para garantir a ressecção total da $\mathrm{VCl}$ acometida. A abordagem transdiafragmática requisitou maior tempo de fase anepática, porém não houve qualquer repercussão hemodinâmica, já que o receptor havia 
desenvolvido anteriormente suficiente retorno venoso através do sistema ázigo. A janela pericárdica foi mantida aberta a fim de evitar tamponamento cardíaco.

As veias ázigo, hemiázigo, hemiázigo acessório e toracolombares desempenham naturalmente uma drenagem colateral à $\mathrm{VCl}$ e têm capacidade notável de desenvolvimento, podendo até suprir a drenagem dos rins e dos membros inferiores. ${ }^{10}$ Desse modo, em casos de obstrução crônica da $\mathrm{VCl}$, a reconstrução do fluxo da veia cava infra-hepática não se fez necessária durante o $\mathrm{TxH}$, devido ao eficiente retorno promovido por esse sistema colateral "natural". ${ }^{11}$ Desse modo, a técnica convencional de $\mathrm{TxH}$ no paciente portador da SBC com sistema de colaterais bem desenvolvido assemelhouse, hemodinamicamente, à técnica Piggyback.

Em contraste, a reconstrução da $\mathrm{VCl}$ deve ser realizada em situações onde não haja drenagem das veias renais e ilíacas diretamente para a veia cava superior, através do sistema ázigo. Ressecção por malignidade em que $\mathrm{VCI}$ não estava anteriormente obstruída ou em pacientes com lesões obstrutivas agudas, sem colaterais venosas bem desenvolvidas são casos que demandam esse tipo de abordagem..$^{12}$ Vale salientar, portanto, o trabalho bem sucedido de Chen et al, que realizaram nove $\mathrm{TxH}$, sendo seis por SBC, com anastomose átrio atrial direta via transdiafragmática com reposição da $\mathrm{VCl}$ com enxerto venoso. $^{13}$

Além disso, a revascularização portal precisou ser modificada no caso presente. O aumento da pressão nos capilares sinusoidais provoca um decréscimo no fluxo portal, o que pode culminar em trombose da veia porta nos pacientes portadores da SBC. ${ }^{14}$ Constatouse no exame de imagem ausência de fluxo na veia porta. Durante a abordagem cirúrgica ficou evidente que havia atrofia da veia devido à trombose venosa portal crônica. Portanto, foi necessário abordar a veia gástrica esquerda, uma colateral bastante desenvolvida na paciente, a fim de restabelecer o suprimento portal do enxerto.

De fato, o maior desafio da cirurgia voltou-se à reconstrução da drenagem venosa das veias hepáticas. Vários métodos têm possibilitado a realização do $\mathrm{TxH}$ nesse grupo de pacientes. No caso de TxH com doadores vivos, a anastomose da veia hepática do enxerto diretamente no átrio direito, pôde ser realizada com bons resultados. ${ }^{11,15}$ Pode-se estabelecer, também, ampla abertura triangular na $\mathrm{VCl}$ ocluída e a realização de uma anastomose que minimize o risco de recorrência da doença. ${ }^{16}$ Há relatos da utilização de enxertos vasculares aórticos para substituir $\mathrm{VCl}$ suprahepática. ${ }^{17}$

\section{CONCLUSÃO}

O TxH é uma terapêutica viável para SBC e pode ser realizado mesmo que haja fibrose intensa e trombose desde o átrio direito até a $\mathrm{VCI}$ infra-hepática, sem a realização de esternotomia mediana. A abordagem transdiafragmática e anastomose cavo-atrial mostra-se como alternativa factível na literatura atual.

A circulação colateral desenvolvida pelo paciente com SBC deve ser avaliada adequadamente antes do $\mathrm{TxH}$, tanto do ponto de vista da $\mathrm{VCl}$, através do sistema ázigo, quanto da veia porta, para determinar a melhor opção de reconstrução vascular.

\section{ABSTRACT}

Introduction: Budd-Chiari is a heterogeneous syndrome of non-specific etiology, characterized by obstruction of hepatic venous outflow at the level of the hepatic veins, up to the right atrium. In those patients, fibrosis of unknown etiology is frequently found between diaphragm and suprahepatic vena cava, making dissection and anastomoses unfeasible in this segment. Purpose: To report and review surgical technique challenges regarding the transdiaphragmatic approach and cavoatrial anastomosis in liver transplantation due to complete occlusion of the retrohepatic inferior vena cava. Method: Case report based on surgical observations, clinical records and images produced during follow-up of the patient in the transplantation service. Data from literature on Pubmed platform supported the discussion. Conclusions: Transdiaphragmatic approach and cavoatrial anastomosis are shown as a feasible alternative in current literature for Budd-Chiari syndrome patients needing transplantation. Replacement of the infrahepatic vena cava depends on development of collaterals through the azygous system and must be assessed during the preoperative period.

Keywords: Liver Transplantation; Budd-Chiari Syndrome; Vena Cava, Inferior. 
Olival Cirilo Lucena da Fonseca Neto; Luiz, Eduardo Rafael Moutinho; Priscylla Jennie Monteiro Rabêlo; Paulo Sergio Vieira de Melo; Américo Gusmão Amorim; Claudio Moura Lacerda

\section{REFERÊNCIAS}

1. Janssen HLA, Garcia-Pagan J-C, Elias E, Mentha G, Hadengue A, Valla D-C, et al. Budd-Chiari syndrome: a review by an expert panel. J Hepatol 2003;38(3):364-71.

2. Menon KVN, Shah V, Kamath PS. The Budd-Chiari syndrome. N Engl J Med 2004;350(6):578-85.

3. Terasaki M, Kitai T, Morimoto T, Kumada K, Sasaki H, Nakano $M$, et al. Hemodynamics and hepatic energy metabolism in canine model of acute hepatic venous occlusion with mesocaval shunt. Eur Surg Res 1994;26(1):19-27.

4. Burtch GD, Merion RM. Transdiaphragmatic exposure for direct atrial-caval anastomosis in liver transplantation for Budd-Chiari syndrome. Transplantation 1989;48(1):161-3.

5. Valla D-C. Budd-Chiari syndrome/hepatic venous outflow tract obstruction. Hepatol Int 2018;12(Suppl 1):168-80.

6. Mentha G, Giostra E, Majno PE, Bechstein WO, Neuhaus P, O'Grady J, et al. Liver transplantation for Budd-Chiari syndrome: A European study on 248 patients from 51 centres. J Hepatol 2006;44(3):520-8.

7. Segev DL, Nguyen GC, Locke JE, Simpkins CE, Montgomery RA, Maley WR, et al. Twenty years of liver transplantation for Budd-Chiari syndrome: a national registry analysis. Liver Transpl 2007;13(9):1285-94.

8. Pan G-D, Yan L-N, Li B, Lu S-C, Zeng Y, Wen T-F, et al. Liver transplantation for patients with hepatolithiasis. HBPD INT 2005;4(3):345-9.

9. Koch S, Bresson-Hadni S, Miguet J-P, Crumbach J-P, Gillet M, Mantion G-A, et al. Experience of liver transplantation for incurable alveolar echinococcosis: a 45-case European collaborative report. Transplantation 2003;75(6):856-63.

10. Gladstone RJ. Development of the Inferior Vena Cava in the Light of Recent Research, with Especial Reference to Certain Abnormalities, and Current Descriptions of the Ascending Lumbar and Azygos Veins. J Anat 1929;64(Pt 1):70-93.
11. Fukuda A, Ogura Y, Kanazawa H, Mori A, Kawaguchi $\mathrm{M}$, Takada $\mathrm{Y}$, et al. Living donor liver transplantation for Budd-Chiari syndrome with hepatic inferior vena cava obstruction after open pericardial procedures. Surg Today 2013;43(10):1180-4.

12. Bower TC, Nagorney DM, Cherry KJ, Toomey BJ, Hallett JW, Panneton JM, et al. Replacement of the inferior vena cava for malignancy: an update. J Vasc Surg 2000;31(2):270-81.

13. Chen Z-Y, Yan L-N, Zeng Y, Wen T-F, Li B, Zhao J-C, et al. Transdiaphragmatic exposure for direct atrioatrial anastomosis in liver transplantation. Chin Med J 2010;123(24):3515-8.

14. Cazals-Hatem D, Vilgrain V, Genin P, Denninger $M-H$, Durand F, Belghiti J, et al. Arterial and portal circulation and parenchymal changes in Budd-Chiari syndrome: a study in 17 explanted livers. Hepatology 2003;37(3):5109.

15. Sabra TA, Okajima H, Tajima T, Fukumitsu K, Hata K, Yasuchika K, et al. Living donor liver transplantation for adult Budd Chiari syndrome - Resection without replacement of retrohepatic IVC: A case report. Int J Surg Case Rep 2018;42:50-4.

16. Karaca C, Yilmaz C, Ferecov R, lakobadze Z, Kilic K, Caglayan L, et al. Living-Donor Liver Transplantation for Budd-Chiari Syndrome: Case Series. Transplant Proc 2017;49(8):1841-7.

17. Cetinkunar S, Ince V, Ozdemir F, Ersan V, Yaylak F, Unal $B$, et al. Living-Donor Liver Transplantation for BuddChiari Syndrome--Resection and Reconstruction of the Suprahepatic Inferior Vena Cava With the Use of Cadaveric Aortic Allograft: Case Report. Transplant Proc 2015;47(5):1537-9. 